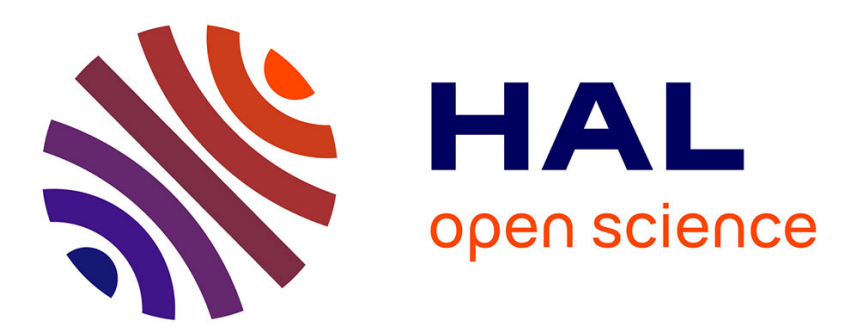

\title{
Prediction of the vibroacoustic behavior of a submerged shell with non-axisymmetric internal substructures by a condensed transfer function method
}

V Meyer, Laurent Maxit, J.-L Guyader, T Leissing

\section{- To cite this version:}

V Meyer, Laurent Maxit, J.-L Guyader, T Leissing. Prediction of the vibroacoustic behavior of a submerged shell with non-axisymmetric internal substructures by a condensed transfer function method. Journal of Sound and Vibration, 2016, 360, pp.260-276. 10.1016/j.jsv.2015.09.030 . hal01272237

\section{HAL Id: hal-01272237 \\ https://hal.science/hal-01272237}

Submitted on 10 Feb 2016

HAL is a multi-disciplinary open access archive for the deposit and dissemination of scientific research documents, whether they are published or not. The documents may come from teaching and research institutions in France or abroad, or from public or private research centers.
L'archive ouverte pluridisciplinaire HAL, est destinée au dépôt et à la diffusion de documents scientifiques de niveau recherche, publiés ou non, émanant des établissements d'enseignement et de recherche français ou étrangers, des laboratoires publics ou privés. 


\title{
Prediction of the vibroacoustic behavior of a submerged shell with non-axisymmetric internal substructures by a Condensed Transfer Function method
}

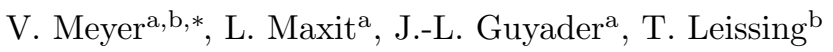 \\ ${ }^{a}$ INSA Lyon, Laboratoire Vibrations Acoustique (LVA), 25 bis av. Jean Capelle, 69210 \\ Villeurbanne, France \\ ${ }^{b}$ DCNS Research, Rond Point de l'Artillerie de Marine, 83000 Toulon, France
}

\begin{abstract}
The vibroacoustic behavior of axisymmetric stiffened shells immersed in water has been intensively studied in the past. On the contrary, little attention has been paid to the modeling of these shells coupled to non-axisymmetric internal frames. Indeed, breaking the axisymmetry couples the circumferential orders of the Fourier series and considerably increases the computational costs. In order to tackle this issue, we propose a sub-structuring approach called the Condensed Transfer Function (CTF) method that will allow assembling a model of axisymmetric stiffened shell with models of non-axisymmetric internal frames. The CTF method is developed in the general case of mechanical subsystems coupled along curves. A set of orthonormal functions called condensation functions, which depend on the curvilinear abscissa along the coupling line, is considered. This set is then used as a basis for approximating and decomposing the displacements and the applied forces at the line junctions. Thanks to the definition and calculation of condensed transfer functions for each uncoupled subsystem and by using the superposition principle for passive linear systems, the behavior of the coupled subsystems can be deduced. A plane plate is considered as a test case to study the convergence of the method with respect to the type and the number of condensation functions taken into account. The CTF method is then applied to couple a submerged non-periodically stiffened shell described using the Circumferential Admittance Approach (CAA) with internal substructures described by Finite Element Method (FEM). The influence of non-axisymmetric internal substructures can finally be studied and it is shown that it tends to increase the radiation efficiency of the shell and can modify the vibrational and acoustic energy distribution.
\end{abstract}

Keywords: Admittance, Sub-structuring, Stiffened shell, Vibroacoustics, Non-axisymmetric, Numerical modeling.

\footnotetext{
* Corresponding author

Email address: valentin.meyer@insa-lyon.fr (V. Meyer)
} 


\section{Introduction}

Modeling the vibroacoustic behavior of submerged stiffened hulls is of primary importance for several industrial applications. The Circumferential Admittance Approach (CAA) [1] is well adapted to describe the vibroacoustic

5 behavior of a submerged shell non periodically stiffened by axisymmetric internal frames. The CAA is a substructuring approach based on the admittance principle for linear systems [2, 3, for which the admittances are defined for each subsystem and for each circumferential order. A dedicated model of the fluid loaded cylindrical shell can then be coupled with axisymmetric Finite Element

10 models of internal frames. It allows modeling the vibroacoustic behavior of the shell with various internal frames (ring stiffeners, axisymmetric bulkheads, end caps) on a wide frequency range, typically from $100 \mathrm{~Hz}$ to $1 \mathrm{kHz}$. However, this method is based on the assumption of an axisymmetric system, so that the circumferential orders can be studied individually. The vibroacoustic behavior of 15 shells with non-axisymmetric internal structures is nevertheless of great interest for naval or aerospace applications. In the present paper, we propose therefore a method called the condensed transfer function (CTF) method to couple an axisymmetric submerged stiffened shell with non-axisymmetric internal frames. This method is based on an extension of the admittance method, allowing to 20 assemble mechanical structures coupled along lines. As the admittances are calculated separately for each subsystem, the CAA model can be used to calculate the admittances of the submerged stiffened shell on one hand. On the other hand, the Finite Element Method (FEM) can be used to calculate the admittances of the non-axisymmetric internal frames.

25 In the past, different models of shell with non-axisymmetric internal structures have been proposed. Some of these studies have focused on the vibroacoustic behavior of non-axisymmetric cylindrical shells in vacuo [4, 5, 6, 7, 8, . Besides, other studies taking into account heavy fluid loading in the shell model can be found. Rebillard et al. 9] add a mass-spring system in an immersed so cylinder. They solve analytically the problem from the eigenmodes of the axisymmetric cylinder in vacuo, include a radiation-impedance term to account for the fluid, and validate the results experimentally. Achenbach, Bjarnason and Igusa [10] study the influence of a non-axisymmetric substructure on a similar configuration and show that the mass-spring system can heftily modify

35 the vibroacoustic behavior of the shell. Their method is based on a variational formulation and the introduction of Lagrange coefficients to account for the coupling forces.Depending on the frequency considered, adding such a subsystem can either increase or decrease the amplitude of the radiated sound field around the cylindrical shell. The same method is used to take into account floors and 40 stiffeners in a 2-D problem on an infinitely long cylindrical shell [11, 12, to highlight the influence of non-axisymmetric excitations [13] and to consider the example of two longitudinal beams lying on stiffeners [14]. In the general formulation, the circumferential orders are coupled and results from the previous studies show that the coupling cannot be neglected in non-axisymmetric prob-

45 lems. This idea reinforces the results given by Laulagnet and Guyader [15, 
who break the axisymmetry of an immersed cylinder through partial masking, namely by overlaying the external surface of the cylinder by an acoustic coating. Similarly, Cuschieri and Feit [16] study the influence of the masking and notice that the coupling of the circumferential modes depends on its regularity.

The increasing complexity of the internal structures, including irregular spacing of stiffeners, non-axisymmetry and the addition of internal degrees of freedom changes some resonances, modifies the acoustic field and couples different modes [17. To tackle these problems, for which geometry is more complex than the academic studies cited above, experimental and numerical methods have 55 been used. Photiadis et al. 18] create an experimental model of an immersed stiffened cylinder with numerous mass-spring systems attached on the stiffeners, and compare the measurements to a model without the resonators. They deduce that the internal structures induce several effects on the mono-static scattering and in particular the attenuation of Bloch-Floquet waves. Bucaro et so al. [19] clarify these results by modifying locally the structure admittance to account for the resonators. Because each complexity level tends to increase the level of the radiated and scattered field, it is advisable for industrial applications to keep as simple systems as possible and not to break the axisymmetry. This conclusion is equivalent to say that discontinuities tend to enhance the ra-

65 diation efficiency of the structures [20. The pressure radiated by an immersed shell with and without point masses is calculated by the Finite Element Method by Marcus and Houston 21. For their example, they show that the coupling of the circumferential modes induces a rise in the radiation level of about 10 $\mathrm{dB}$ over a broad frequency range. Studies by coupled FEM-BEM (Finite Ele70 ment Method - Boundary Element Method) give results at low frequency that highlight the crucial importance of isolating internal structures through flexible mounts [22, 23. Ettouney et al. 24] solve the problem of a submerged axisymmetric shell by using the uncoupled circumferential harmonics. They use a sub-structuring approach based on the admittances to include three-dimensional 75 systems in the axisymmetric shell while keeping the circumferential orders uncoupled. The system is thus equivalent to an axisymmetric submerged shell with internal point forces that account for the presence of the internal systems, but the method is however limited to point coupling.

In general, the element based methods are well suited for modeling systems so with high geometry and property complexity. Due to the current computational limits, they are however limited to low frequencies when the system to be described is a submerged shell. On the contrary, semi-analytical models as described previously are available to analyze the behavior of shells coupled with particular internal non-axisymmetric structures such as oscillators, beams,

85 simply supported plates, etc., over a wide frequency range. The limitations of these methods lie in general on their low versatility, meaning that a dedicated model should be developped for each type of internal structure. The method proposed in this paper consists in benefiting from these different methods, so that the submerged stiffened shell is modeled by the CAA method whereas the go non-axisymmetric internal structures are modeled by FEM, allowing for flexibility with their geometry. The junctions between the stiffened shell and the 
non-axisymmetric internal frames are supposed to be lines. A set of orthonormal functions called condensation functions are used as a basis for approximating the displacements and the forces at the junctions. Condensed transfer functions are then defined and calculated for each uncoupled subsystem, and the superposition principle for passive linear systems leads to the vibroacoustic behavior of the coupled system. The paper is organized as follow:

- In section 2, the principle of the CTF method is described for the general case of subsystems coupled along a line. A set of orthonormal functions called condensation functions is considered to approximate the forces and displacements at the line junctions.

- Three different examples of condensation functions are considered to validate the CTF method on a plane plate case and convergence criteria are studied in section 3 .

- In section 4, the CTF method is applied to add non-axisymmetric internal frames to a stiffened shell. An in vacuo case is first defined and the results are compared with FE calculation to validate the numerical process. Finally, the radial displacements and the pressure radiated by a non-axisymmetric stiffened submerged shell are presented and physical phenomena are discussed.

\section{Principle of the Condensed Transfer Function (CTF) method}

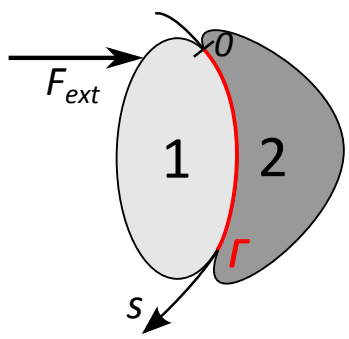

(a)

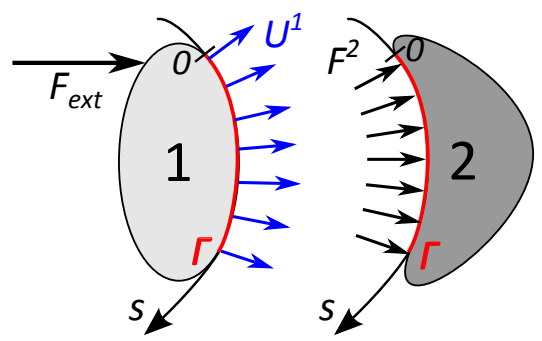

(b)

Figure 1: Admittance method extended to line coupling. (a) Subsystems coupled along a line. (b) Subsystems uncoupled.

Let us consider two mechanical thin structures coupled along a line, as shown in Fig. 1. $\Gamma$ represents the coupling line and the curvilinear abscissa $s$ is used to locate a point on $\Gamma$. A set of $N$ orthonormal functions is considered and are called the condensation functions: $\left\{\varphi_{i}\right\}_{1 \leq i \leq N}$. The type and the number of condensation functions $N$ may play a key role in the convergence of the method and will be discussed later in this paper. One assumes that the displacements $U^{\alpha}(s)$ and the line coupling forces $F^{\alpha}(s)$ at the junction can be approximated for 
each subsystem $\alpha$ as a linear combination of these condensation functions. For each uncoupled subsystem $\alpha \in\{1,2\}$, the condensed transfer function between $\varphi_{i}$ and $\varphi_{j}$ is defined by applying a force $F^{\alpha}=\varphi_{j}$ on $\Gamma$ :

$$
Y_{i j}^{\alpha}=\frac{\left\langle\bar{U}_{j}^{\alpha}, \varphi_{i}\right\rangle}{\left\langle F^{\alpha}, \varphi_{j}\right\rangle}=\left\langle\bar{U}_{j}^{\alpha}, \varphi_{i}\right\rangle
$$

where $\langle\bullet, \bullet\rangle$ is a scalar product and $\bar{U}_{j}^{\alpha}$ the displacement of the junction $\Gamma$ when the subsystem is excited by $F^{\alpha}=\varphi_{j}$.

Moreover, the free condensed displacement of each uncoupled subsystem $\alpha$ 125 is defined by:

$$
\tilde{u}_{i}^{\alpha}=\left\langle\tilde{U}^{\alpha}, \varphi_{i}\right\rangle
$$

where $\tilde{U}^{\alpha}$ is the displacement at the junction of the uncoupled subsystem $\alpha$ when only external loading is applied. As in the example of Fig. 1, the subsystem 2 has no external load: $\tilde{u}_{i}^{2}=0, \forall i \in \llbracket 1, N \rrbracket$. As proposed above, the displacement and the line force applied on subsystem $\alpha$ can be decomposed using the condensation

$$
\left\{\begin{array} { l } 
{ U ^ { 1 } ( s ) \simeq \sum _ { i = 1 } ^ { N } u _ { i } ^ { 1 } \varphi _ { i } ( s ) } \\
{ U ^ { 2 } ( s ) \simeq \sum _ { i = 1 } ^ { N } u _ { i } ^ { 2 } \varphi _ { i } ( s ) }
\end{array} \quad \text { and } \quad \left\{\begin{array}{l}
F^{1}(s) \simeq \sum_{i=1}^{N} f_{i}^{1} \varphi_{i}(s) \\
F^{2}(s) \simeq \sum_{i=1}^{N} f_{i}^{2} \varphi_{i}(s)
\end{array}\right.\right.
$$

where $u_{i}^{\alpha}$ (resp. $f_{i}^{\alpha}$ ) is the displacement amplitude (resp. the force amplitude) of subsystem $\alpha$ associated to the condensation function $\varphi_{i}$. In response to these line coupling forces and the external load, the superposition principle for passive linear systems enables to write the displacements coefficients $u_{i}^{\alpha}$ as follows:

$$
\left\{\begin{array}{c}
u_{i}^{1}=\tilde{u}_{i}^{1}+\sum_{j=1}^{N} Y_{i j}^{1} f_{j}^{1} \\
u_{i}^{2}=\sum_{j=1}^{N} Y_{i j}^{2} f_{j}^{2}
\end{array} \quad, \forall s \in \Gamma, \forall i \in \llbracket 1 ; N \rrbracket\right.
$$

Besides, the displacement continuity and force equilibrium at the junction lead to:

$$
\left\{\begin{array}{c}
U^{1}(s)=U^{2}(s) \\
F^{1}(s)+F^{2}(s)=0
\end{array}, \forall s \in \Gamma\right.
$$

The set of condensation functions being orthonormal, the projection of Eq. (5) on a function $\varphi_{i}$ yields:

$$
\left\{\begin{array}{c}
u_{i}^{1}=u_{i}^{2} \\
f_{i}^{1}=-f_{i}^{2}
\end{array}, \forall i \in \llbracket 1 ; N \rrbracket\right.
$$

Injecting Eq. (4) in Eq. (6) results in a similar formula compared to the 140 classical admittance method to deduce the coupling forces $\mathbf{F}^{\mathbf{c}}=\mathbf{F}^{\mathbf{1}}=-\mathbf{F}^{\mathbf{2}}$ between the subsystems:

$$
\left(\mathbf{Y}^{1}+\mathbf{Y}^{2}\right) \mathbf{F}^{\mathbf{c}}=-\tilde{\mathbf{U}}^{1}
$$


In the general case, for a given subsystem $\alpha$ and a couple of condensation functions $\left(\varphi_{i}, \varphi_{j}\right)$, the matrix $\mathbf{Y}^{\alpha}$ is a square matrix which size depends on the number of degrees of freedom (generally 6 in 3 -D problems), to take into account all the possible directions of excitations and displacements. Thus, the matrix $\mathbf{Y}^{\alpha}$ has $6 N \times 6 N$ elements. Similarly, $\tilde{\mathbf{U}}^{\alpha}$ is a $6 N$ elements vector (still considering having a general 3-D problem) to calculate the response of the edge in all directions of space to the external load. The size of this system is therefore equal to the number of transfer functions multiplied by the number of degrees

\section{Numerical study on a basic test case}

\subsection{Test case parameters}

To validate and study the numerical convergence of the CTF approach, let having the same thickness. The aim is to couple the two plates together along their longest edge, as shown in Fig. 2. The characteristics and dimensions are given in Table 1 . The plates lie in the $z=0$ plane and the boundary conditions are free on all the edges. Considering the coordinates origin at the bottom left

180 of plate 1 , the system is excited by a transverse harmonic excitation on plate 1 at the point with the coordinates $(0.9,0.5)$. 

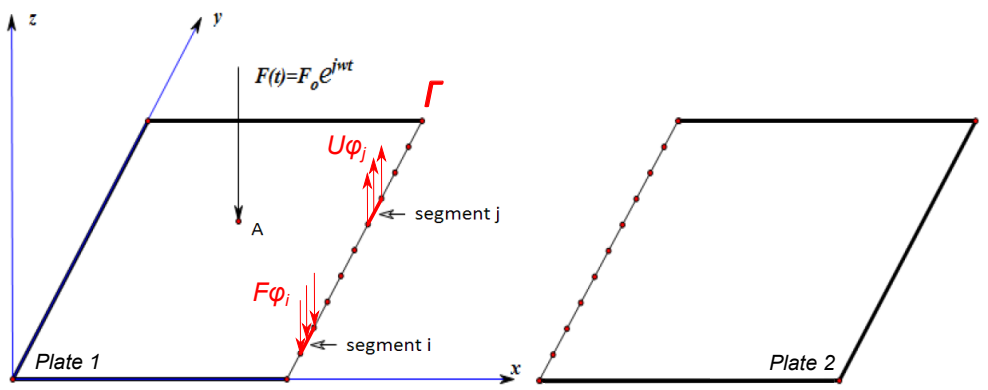

Figure 2: Two rectangular plates coupled along a line. Partition of the coupling line into 10 segments.

\begin{tabular}{cccc}
\hline Parameter & Notation & Value & Unit \\
\hline Young modulus & $E$ & 210 & $\mathrm{GPa}$ \\
Poisson coefficient & $\nu$ & 0.3 & - \\
Density & $\rho_{p}$ & 7800 & $\mathrm{~kg} \cdot \mathrm{m}^{-3}$ \\
Structural damping coefficient & $\eta$ & 0.02 & - \\
Length of plate 1 \& 2 & $L$ & 1.5 & $\mathrm{~m}$ \\
Width of plate 1 & $l_{1}$ & 1.2 & $\mathrm{~m}$ \\
Width of plate 2 & $l_{2}$ & 1.3 & $\mathrm{~m}$ \\
Thickness & $h$ & 0.017 & $\mathrm{~m}$ \\
\hline
\end{tabular}

Table 1: Material characteristics and plates dimensions. 
For comparison purposes, a reference calculation is made using the FEM to model a $1.5 \times 2.5 \mathrm{~m}^{2}$ plate. Harmonic responses are calculated for frequencies between $10 \mathrm{~Hz}$ and $1500 \mathrm{~Hz}$, with approximately 400 values logarithmically spread over the domain. This frequency resolution ensures to properly describe the resonance peaks of the system regarding the value of the structural damping coefficient [27, which is accounted for as a complex factor in the stiffness matrix. One layer of quadrilateral isotropic shell elements of thickness $h$ is chosen with a linear interpolation function. The Finite Element mesh is fine enough 190 in order to respect the criterion of 6 elements per bending wavelength which is commonly used for such problems.

To apply the CTF approach, the condensed admittances should be calculated for the two subsystems. As discussed previously, the estimation of the response of each uncoupled subsystem excited on the junction line by forces corresponding to condensation functions are needed. FEM calculations and the modal superposition including quasi-static residual modes 28] can be used for this purpose thanks to its good convergence. The principle of this technique is presented in Appendix A. The modal basis is truncated such that the frequency of the highest mode is 1.9 times higher than the highest frequency. In the case

200 of the two plates described previously, the basis for plate 1 counts 116 modes (highest eigenfrequency at $f=2848.6 \mathrm{~Hz}$ ) and 125 modes for plate 2 (highest eigenfrequency at $f=2839.3 \mathrm{~Hz}$ ). Three examples of condensation functions are given in the next subsection before discussing the results for the present test case.

\subsection{Three examples of condensation functions}

\subsubsection{Gate functions}

The gate functions $\varphi_{i}, i \in \llbracket 1, N \rrbracket$, are defined depending on their length $L_{s}$ as follows:

$$
\varphi_{i}(s)= \begin{cases}\frac{1}{\sqrt{L_{s}}} & \text { if }(i-1) L_{s} \leq s<i L_{s} \\ 0 & \text { elsewhere }\end{cases}
$$

As the junction $\Gamma$ has a finite length, giving a gate length $L_{s}$ leads to a number $N$ of condensation functions taken into account. The scalar product associated with the gate function is defined by:

$$
\begin{aligned}
\mathcal{C}_{I}^{0} \times \mathcal{C}_{I}^{0} & \rightarrow \mathbb{C} \\
\langle f, g\rangle & \mapsto \int_{\Gamma} f(s) g^{*}(s) \mathrm{d} s
\end{aligned}
$$

where $\mathcal{C}_{I}^{0}$ is the set of piecewise continuous functions on intervals $\left[a, b\left[\subset \Gamma\right.\right.$, and ${ }^{*}$ means the complex conjugate. It can be easily verified that the gate functions form an orthonormal set for this scalar product.

In this case, applying the CTF approach can be seen as dividing the coupling line into $N$ segments and calculating the admittances between the segments, as shown in Fig. 2 The segments are excited one after the other, and the displacement on each segment allows to obtain the admittance matrix of each 
uncoupled subsystem. Each term of the free displacement vector is the result of the integral of the displacement on one segment due to the external load (only for plate 1 in this example).

\subsubsection{Exponential functions}

Complex exponential functions can be well adapted to describe the flexural waves at the junction. These exponential functions are defined by:

$$
\varphi_{i}(s)=\frac{1}{\sqrt{L}} \exp \left(\mathrm{j} \frac{i \pi s}{L}\right)
$$

${ }_{225}$ where $L$ is the length of the junction $\Gamma$ and $\mathrm{j}$ the complex number $\mathrm{j}^{2}=-1$. The associated scalar product is defined as follows, ensuring the set to be orthonormal:

$$
\begin{aligned}
\mathcal{C}^{0} \times \mathcal{C}^{0} & \rightarrow \mathbb{C} \\
\langle f, g\rangle & \mapsto \int_{\Gamma} f(s) g^{*}(s) \mathrm{d} s
\end{aligned}
$$

where $\mathcal{C}^{0}$ is the set of continuous function on $\Gamma$. The set of condensation functions is characterized by the maximal index $i_{\max }$ and all the functions such that $i \in \llbracket-i_{\max }, i_{\max } \rrbracket$ are taken into account.

\subsubsection{Chebyshev polynomials}

The Chebyshev polynomials are widely used to interpolate functions and are thus an interesting alternative for the condensation functions [29. They can be defined recursively by the following relations:

$$
\left\{\begin{aligned}
T_{0}(X) & =1 \\
T_{1}(X) & =X \\
T_{n+2}(X) & =2 X T_{n+1}(X)-T_{n}(X)
\end{aligned}\right.
$$

The scalar product associated to the Chebyshev polynomials takes into account the weight function $\frac{1}{\sqrt{s(L-s)}}$ :

$$
\begin{aligned}
\mathcal{C}^{0} \times \mathcal{C}^{0} & \rightarrow \mathbb{C} \\
\langle f, g\rangle & \mapsto \frac{L}{\pi} \int_{\Gamma} f(s) g^{*}(s) \frac{1}{\sqrt{s(L-s)}} \mathrm{d} s
\end{aligned}
$$

The weight function ensures the set $\frac{T_{0}}{\sqrt{2}} \cup\left(T_{n}\right)_{n \in \mathbb{N}^{*}}$ to be orthonormal in regards to this scalar product and reduces numerical errors by quadratically clustering the nodes at the end of the segment.

\subsection{Results}

The mean (average on surfaces) quadratic transversal velocity on the surface of the two plates coupled together is calculated using the CTF method with 
the 3 different condensation functions presented above and is compared to a reference solution. The responses and the relative errors are plotted in Fig. 3 as 245 a function of the frequency. The CTF calculations have been performed taking 3 condensation functions $(N=3)$, which means:

- dividing the line junction in 3 segments when the condensation functions are gates;

- taking $i_{\max }=1$ for the exponential functions, i.e.:

$$
\varphi(s) \in\left\{\frac{1}{\sqrt{L}} \exp \left(\frac{-\mathrm{j} \pi s}{L}\right) ; \frac{1}{\sqrt{L}} ; \frac{1}{\sqrt{L}} \exp \left(\frac{\mathrm{j} \pi s}{L}\right)\right\}
$$

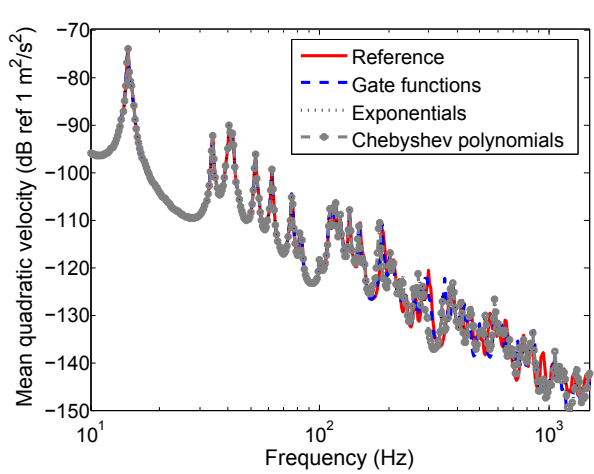

(a)

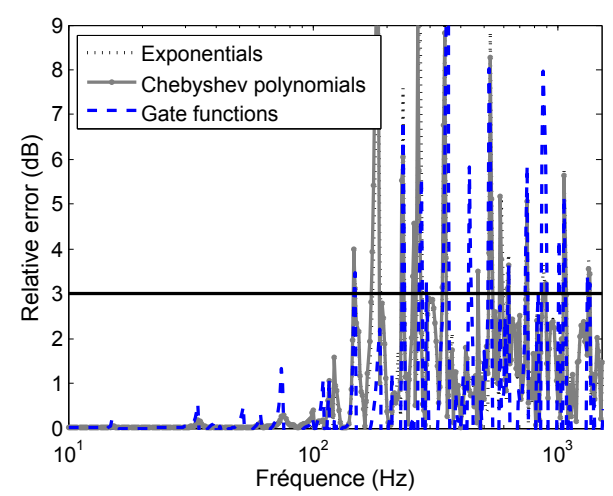

(b)

Figure 3: Spatial averaged quadratic transversal velocity on the two coupled plates and excited by a point harmonic force. Comparison between the reference and the CTF methods with $N=3$. (a) Narrow band response. (b) Relative error (dB).

One can observe that all the results agree well until around $f_{\text {lim }} \simeq 150$ $\mathrm{Hz}$. For this test case, some differences up to $1 \mathrm{~dB}$ can be seen at the resonance frequencies in the case of the gate functions. These differences are due to a slight frequency shift in the resonance and can be acceptable for practical applications. Above this frequency, the transfer functions are not able to describe properly the phenomena. In other terms, the forces and displacements cannot be correctly interpolated by the 3 condensation functions at higher frequencies.

\subsection{Convergence criteria}

Assuming that at least 2 points per wavelength are necessary to sample a signal ( $c f$. Nyquist-Shannon sampling theorem), one can propose criteria defining the characteristics of the set of condensation functions $\left(L_{s}, i_{\max }, N_{\max }\right)$ as a function of the flexural wavelength $\lambda_{f}$ of the plate at the higher frequency limit $f_{\text {lim }}$ : 
- for gate functions, two segments are needed to describe a wavelength:

$$
L_{S} \leq \frac{\lambda_{f}}{2}
$$

- for exponential functions, noting that the index $i$ can take negative values, the criterion is:

$$
i_{\max } \geq \frac{L_{y}}{\lambda_{f}}-\frac{1}{2}
$$

- for Chebyshev polynomials, the degree of the polynomial must be at least equal to the number of points that the function needs to interpolate:

$$
N_{\max } \geq \frac{2 L_{y}}{\lambda_{f}}-1
$$

These criteria are adapted for each type of condensation function, but give the same number of transfer functions for a given frequency limit. In the case of a $17 \mathrm{~mm}$ thick plate, $N=3$ leads to a frequency limit of $f_{\text {lim }}=168 \mathrm{~Hz}$, which fits the observations in Fig. 3 .

Applying the criterion for Chebyshev polynomials, the convergence of the results is ensured up to more than $1500 \mathrm{~Hz}$ with $N=10$ transfer functions, as seen in Fig. 4 a. For the sake of succinctness, the results of the CTF method with 10 transfer functions with the gate functions and the complex exponentials are not plotted here but shows also good convergence. At this stage of the study, it cannot be said if one of the three condensation functions perform best. The gate functions however present the advantages of being easy to implement and of having a lower condition number, being thus less sensitive to numerical errors 30. In Fig. 4 4 , the amplitude of the transverse force is plotted as a function of the frequency and the condensation functions. For each frequency, the values are normalized with regard to the highest amplitude. Considering the dispersion relation for a plate, Eq. (17) yields :

$$
N_{\max } \geq \sqrt{\frac{2 f}{\pi}} L_{y}\left(\frac{E h^{2}}{12\left(1-\nu^{2}\right) \rho_{p}}\right)^{1 / 4}-1
$$

$N_{\max }$ is an integer and rounded toward positive infinity. $N_{\max }$ is plotted as a function of the frequency in Fig. $4 \mathrm{~b}$ using a white line. It clearly shows that the higher the frequency, the more Chebychev polynomials play a role in the method. It also shows that, except for some frequency values below $55 \mathrm{~Hz}$, the criterion is very well suited to properly approximate the forces at the junction.

\section{Cylindrical shell with non-axisymmetric internal frames}

In order to validate the method for our case of interest and to illustrate its advantages in coupling different models, it is applied for the case of an axisymmetric stiffened shell coupled with non-axisymmetric internal structures. First, 


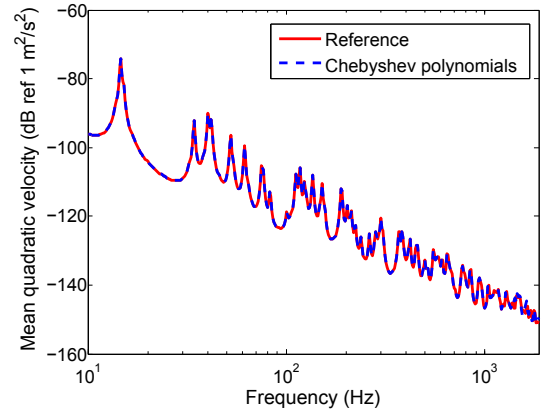

(a)

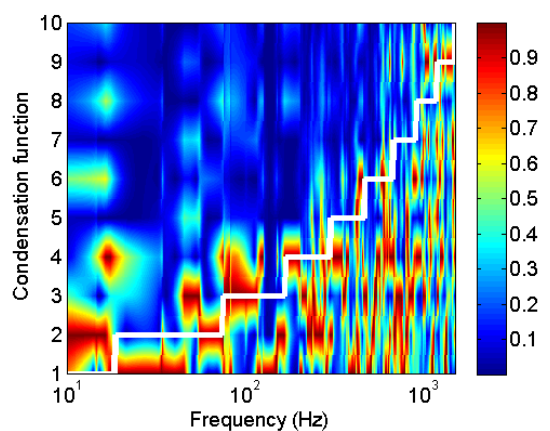

(b)

Figure 4: CTF method with $N=10$ Chebychev polynomials. (a) Mean quadratic velocity as a function of the frequency. (b) Normalized amplitude of the transverse force at the junction projected on the condensation functions as a function of the frequency.

the principle of the approach is described for the general case in subsection 4.1

before highlighting the procedure to calculate the condensed transfer functions from a dedicated CAA model [1] in subsection 4.2. Then, for validation purpose, an in vacuo shell is considered, so that results can be easily compared with a full FEM calculation. Finally, the method is applied to an example of submerged stiffened shell with non-axisymmetric internal structures to discuss the influence of the non-axisymmetric structures on the vibroacoustic behavior.

\subsection{Principle of the CTF approach in the case of a cylindrical shell}

Let us consider a stiffened cylindrical shell with a non-axisymmetric internal frame, as shown on the sketch in Fig. 5. The junctions between the two subsystems are arcs at the ends of the ring stiffeners flange, extending between $\theta_{0}$ and $\theta_{N+1}$.

The Condensed Transfer Function method presented in the previous section enables to take into account non-axisymmetric internal frames in an immersed stiffened shell, as shown on the diagram in Fig. 6. On one hand, the admittances of the axisymmetric submerged stiffened shell are estimated by the CAA. During

310 this step, a numerical model of an immersed shell is coupled with Finite Element models of stiffeners and bulkheads to calculate the admittance terms needed as an input of the CTF method. On the other hand, the admittances of the nonaxisymmetric internal frames are calculated by FEM. Finally, Eq. 7 and 8 are used to deduce the shell displacements and the radiated pressure field.

\subsection{Calculation of the Condensed Transfer Functions of an axisymmetric stiff-} ened shell by the Circumferential Admittance Approach (CAA)

In this part, the Circumferential Admittance Approach (CAA) [1] is used for modeling the vibroacoustic behavior of a non periodically stiffened submerged shell, and to calculate the admittances needed as an input for the CTF approach. 


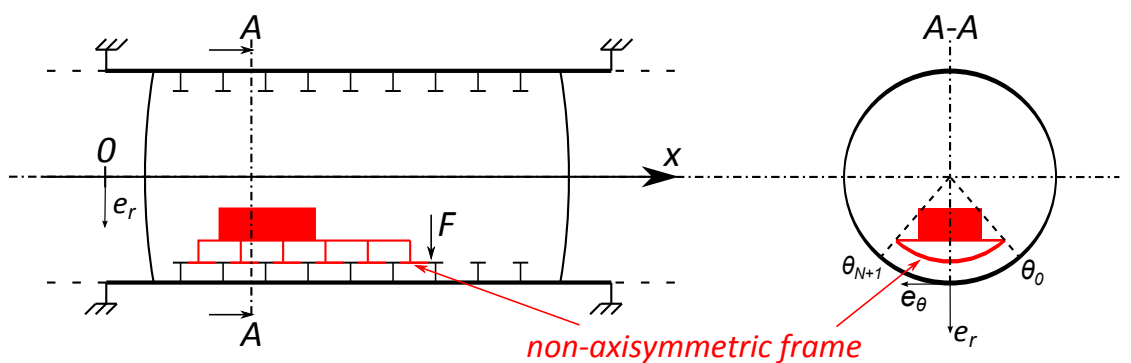

(a)

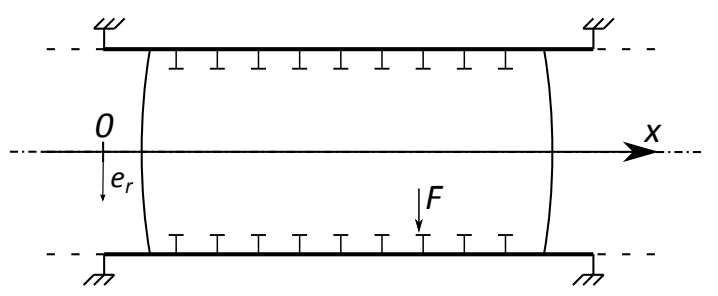

(b)

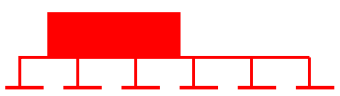

(c)

Figure 5: Sections of a stiffened cylindrical shell. (a) Including a non-axisymmetric internal frame. (b) Axisymmetric partition described using CAA. (c) Example of non-axisymmetric internal frame.

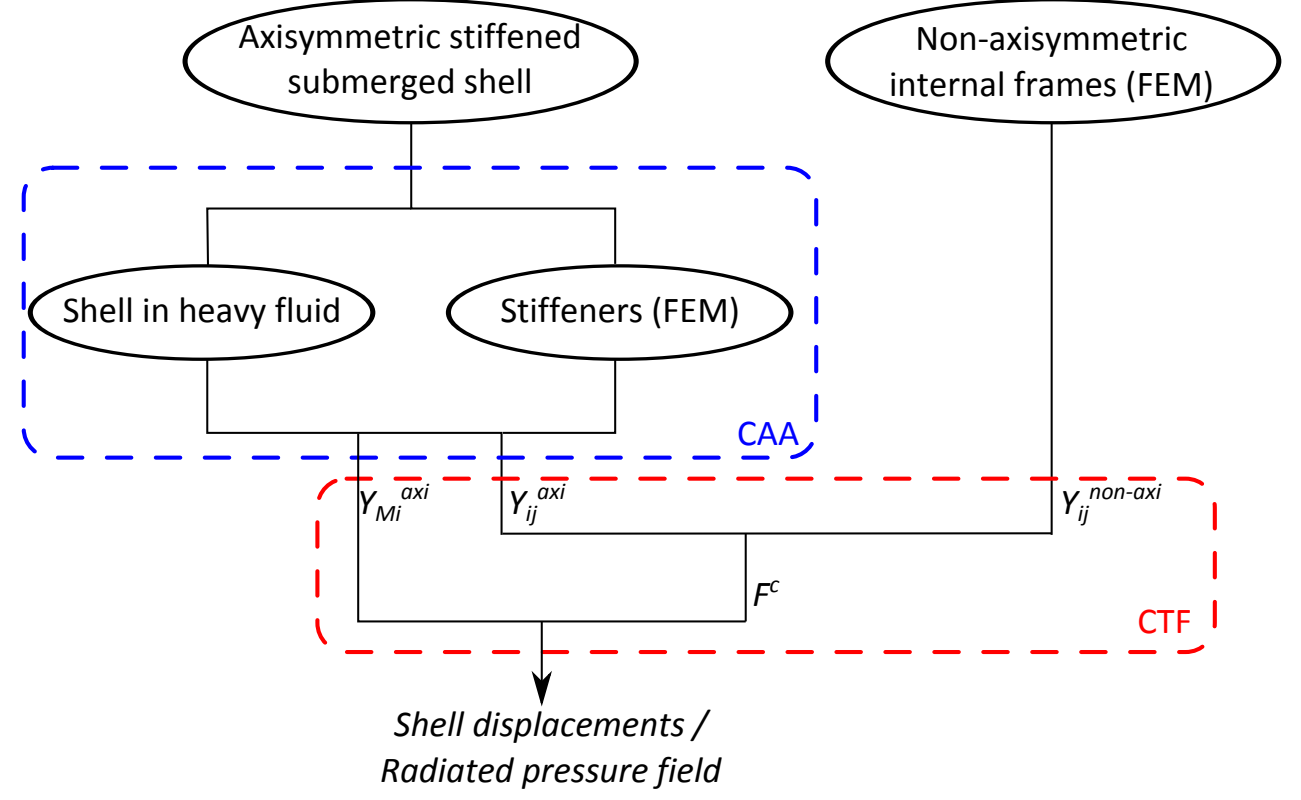

Figure 6: Sketch of the method to account for non-axisymmetric internal frames in a non periodically stiffened submerged shell. 
320 The CAA consists in partitioning the system and considering the submerged shell separately from the internal frames (ring stiffeners, bulkheads, end caps). Considering cylindrical coordinates $(x, r, \theta)$, as shown in Fig. 5, all the physical variables (i.e. forces, shell displacements, pressure,...) can be written as Fourier series depending on circumferential orders $k_{\theta}$ :

$$
f(\theta)=\sum_{k \in \mathbb{Z}} \tilde{f}\left(k_{\theta}\right) \mathrm{e}^{\mathrm{j} k_{\theta} \theta}
$$

where $k_{\theta} \in \mathbb{Z}$, because the system is $2 \pi$-periodic along the circumference, and with

$$
\tilde{f}\left(k_{\theta}\right)=\frac{1}{2 \pi} \int_{-\pi}^{\pi} f(\theta) \mathrm{e}^{-\mathrm{j} k_{\theta} \theta} \mathrm{d} \theta
$$

In this approach, the shell and the frames are axisymmetric and the fluid domain is infinite. Under these assumptions, the circumferential orders are independent from each other [31].

On one hand, the circumferential admittances of the fluid loaded shell are estimated using the Flügge equations of motions [32, 33] and by solving the problem in the wavenumber space. The resolution of these equations are performed using an accelerated spectral approach, as it has proven very good results in terms of accuracy and calculation costs. On the other hand, different types of 335 internal frames can be modeled using axisymmetric FEM. The coupling forces between the shell and the internal frames are then calculated using the circumferential admittances of the subsystems. The forces are finally injected in the shell model to deduce the shell displacements and the radiated pressure. Details on this method can be found in 1 .

340 In order to use the CTF approach to add a non-axisymmetric internal frame, two quantities are needed from the CAA: the condensed transfer functions $Y_{i j}^{a x i}$ at the junctions with the non-axisymmetric system, and the transfer function $Y_{M i}^{a x i}$ between a point $M$ and a condensation function $\varphi_{i}$ applied on one of the junctions, in order to use Eq. (8) in the last step of the CTF calculation. If $M$

345 is a point on the cylindrical shell surface, the latter transfer function represents the displacement of the point when the axisymmetric shell is excited by the condensation function $\varphi_{i}$. If $M$ is a point in the fluid domain outside the shell, $Y_{M i}^{a x i}$ represents the pressure at the point when the axisymmetric shell is excited by the condensation function $\varphi_{i}$. As the system is axisymmetric, the response (displacement or pressure) of any point $M$ with the coordinates $\left(x_{M}, r_{M}, \theta_{M}\right)$ in response to a point harmonic force on the structure at a point $A$ with the coordinates $\left(x_{A}, r_{A}, \theta_{A}\right)$ yields the following admittances:

$$
Y_{M A}^{a x i}=Y_{M^{\prime} A^{\prime}}^{a x i}
$$

where $M^{\prime}$ is the point with the coordinates $\left(x_{M}, r_{M}, \theta_{M}-\theta_{A}\right)$ and $A^{\prime}$ is the point with the coordinates $\left(x_{A}, r_{A}, 0\right)$. As the CAA enables to calculate any $355 Y_{M A}^{a x i}$, let us then consider for the sake of clarity that $\theta_{A}=0$. In the following, 
$Y^{a x i}$ is written as a function of $\theta$ meaning that it is the value at the point of coordinate $\theta$ in response to an excitation in $\theta=0$.

The displacement $\bar{U}_{j}(\theta)$ of a point of coordinate $\theta$ in response to a condensed force $\varphi_{j}$ is then calculated by convolution:

$$
\bar{U}_{j}(\theta)=\int_{\theta_{0}}^{\theta_{N+1}} Y^{a x i}(\theta-\alpha) \varphi_{j}(\alpha) \mathrm{d} \alpha
$$

\subsection{Validation of an in vacuo shell}

\subsubsection{Definition of the system}

A cylindrical shell of radius $R=5 \mathrm{~m}$, length $L=4 \mathrm{~m}$ and thickness $e=$ $30 \mathrm{~mm}$ is clamped at both ends. It is stiffened by two identical stiffeners with 370 $\mathrm{T}$ cross-section at $x=1$ and $x=2 \mathrm{~m}$ from the boundary of the shell. A Tstiffener consists of two perpendicular parts called the flange and the web, the web being the one in the $(O y z)$ plane (see Fig. 7). The web is characterized by its height $h_{w}$ and thickness $t_{w}$ and the flange is characterized by its width $w_{f}$ and thickness $t_{f}$. Both the web and the flange are modeled by shell elements.

375 In this example, the T-stiffeners have the following dimensions: $\left(h_{w} \times t_{w} / w_{f} \times\right.$ $\left.t_{f}\right)=(200 \times 15 / 200 \times 15 \mathrm{~mm})$. A curved plate with a curvature radius of $R_{\text {int }}=4.8 \mathrm{~m}$ links the two stiffeners as shown in Fig. 77. The shell along with the two stiffeners will be referred to as the axisymmetric subsystem, whereas the plate is the non-axisymmetric subsystem, extending between $\theta_{0}$ and $\theta_{N+1}$, ${ }_{380}$ with $-\theta_{0}=\theta_{N+1}=0.2083 \mathrm{rad}$. The dimension of the plate is thus $0.8 \times 2 \mathrm{~m}^{2}$ and it is $15 \mathrm{~mm}$ thick. An unitary external load is applied at the joint between the first stiffener and the plate at $\theta=0$. The shell is not immersed in heavy fluid so that a reference calculation can easily be done by FEM. 


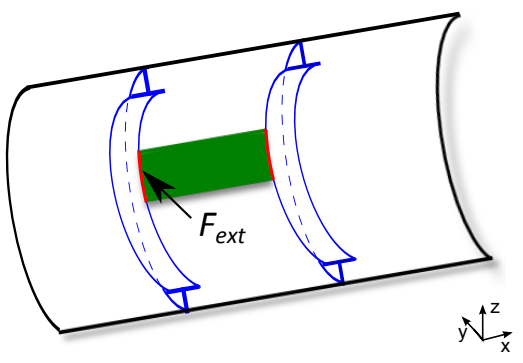

(a)

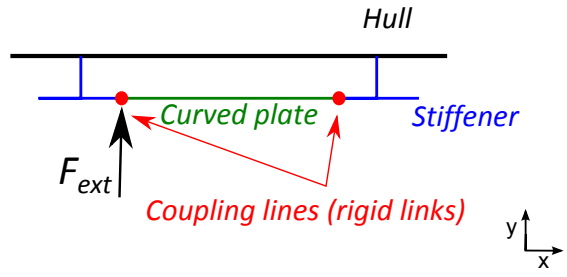

(b)

Figure 7: Cylindrical stiffened shell with a non-axisymmetric internal structure. (a) 3D-view of a section. (b) Section in the plane $\theta=0$.

\subsubsection{CTF calculations} described in 3.2.1. Indeed, among the three condensation functions tested, the gate functions are the easiest to implement. Their length match the criterion proposed in Eq. (15) up to $1 \mathrm{kHz}$. At this frequency, the flexural wavelength of a $15 \mathrm{~mm}$ thick plate is $\lambda_{f}=0.38 \mathrm{~m}$. As the curvature increases the stiffness of the plate, the wavelength at a given frequency increases as well, and the criterion is more restrictive than it could be. The junction is on a circular arc of radius $4.8 \mathrm{~m}$. Thus, 10 gates per junction, of angular length $\theta_{s}=0.0417 \mathrm{rad}$ (equivalent to a curvilinear length of $L_{s}=0.2 \mathrm{~m}$ ) are considered to ensure good convergence up to $1 \mathrm{kHz}$.

In this configuration, the gates are functions of $\theta\left(\varphi_{i}(\theta)=1\right.$ between $\theta_{1}^{i}$ and $\theta_{2}^{i}$, and 0 elsewhere). Writing the terms $Y^{a x i}$ as Fourier series, Eq. (23) leads to the admittance $Y_{i j}^{a x i}$ of the axisymmetric system in the physical space:

$$
\begin{aligned}
Y_{i j}^{a x i}= & \frac{1}{\sqrt{\theta_{2}^{i}-\theta_{1}^{i}}} \int_{\theta_{1}^{i}}^{\theta_{2}^{i}}\left(\frac{1}{\sqrt{\theta_{2}^{j}-\theta_{1}^{j}}} \int_{\theta_{1}^{j}}^{\theta_{2}^{j}} \sum_{k_{\theta}} \tilde{Y}^{a x i}\left(k_{\theta}\right) \mathrm{e}^{\mathrm{j} k_{\theta}(\theta-\alpha)} \mathrm{d} \alpha\right) \mathrm{d} \theta \\
= & \sqrt{\theta_{2}^{j}-\theta_{1}^{j}} \sqrt{\theta_{2}^{i}-\theta_{1}^{i}} \tilde{Y}^{a x i}(0) \\
& +\frac{1}{\sqrt{\theta_{2}^{i}-\theta_{1}^{i}}} \frac{1}{\sqrt{\theta_{2}^{j}-\theta_{1}^{j}}} \sum_{k_{\theta} \neq 0} \frac{1}{k_{\theta}^{2}} \tilde{Y}^{a x i}\left(k_{\theta}\right)\left(\mathrm{e}^{-\mathrm{j} k_{\theta} \theta_{2}^{j}}-\mathrm{e}^{-\mathrm{j} k_{\theta} \theta_{1}^{j}}\right)\left(\mathrm{e}^{\mathrm{j} k_{\theta} \theta_{2}^{i}}-\mathrm{e}^{\mathrm{j} k_{\theta} \theta_{1}^{i}}\right)
\end{aligned}
$$

with $\tilde{Y}^{a x i}$ the projection of the admittance on the circumferential orders. Similarly, Eq. (24) yields:

$$
Y_{M j}^{a x i}=\frac{1}{\sqrt{\theta_{2}^{j}-\theta_{1}^{j}}} \int_{\theta_{1}^{j}}^{\theta_{2}^{j}} \sum_{k_{\theta}} \tilde{Y}^{a x i}\left(k_{\theta}\right) \mathrm{e}^{\mathrm{j} k_{\theta}(\theta-\alpha)} \mathrm{d} \alpha
$$




$$
=\sqrt{\theta_{2}^{j}-\theta_{1}^{j}} \tilde{Y}^{a x i}(0)+\frac{\mathrm{j}}{\sqrt{\theta_{2}^{j}-\theta_{1}^{j}}} \sum_{k_{\theta} \neq 0} \frac{1}{k_{\theta}} \tilde{Y}^{a x i}\left(k_{\theta}\right)\left(\mathrm{e}^{-\mathrm{j} k_{\theta} \theta_{2}^{j}}-\mathrm{e}^{-\mathrm{j} k_{\theta} \theta_{1}^{j}}\right) \mathrm{e}^{\mathrm{j} k_{\theta} \theta}
$$

${ }_{400}$ In practice, Eq. (26) and (27) are truncated to a maximal circumferential order $\bar{N}$. This number is defined considering the natural wavenumbers of the shell, the frames and the acoustic fluid, as explained in 11.

On the other hand, the non-axisymmetric plate is modeled by FEM. The responses are estimated by modal superposition including residual modes (see Appendix A and its condensed transfer functions are calculated by projection on the condensation functions.

\subsubsection{Results}

Equation (7) gives the condensed coupling forces $\mathbf{F}^{\mathbf{c}}$ between the plate and the axisymmetric system. According to Eq. (8), the pressure $P$ at a point $M_{1}$ in ${ }_{410}$ the fluid domain and the displacement $U$ at a point $M_{2}$ on the shell are finally given by:

$$
\left\{\begin{array}{l}
P\left(M_{1}\right)=\tilde{P}\left(M_{1}\right)+\sum_{i=1}^{N} Y_{M_{1} i}^{a x i} F_{i}^{c} \\
U\left(M_{2}\right)=\tilde{U}\left(M_{2}\right)+\sum_{i=1}^{N} Y_{M_{2} i}^{a x i} F_{i}^{c}
\end{array}\right.
$$

where $\tilde{P}\left(M_{1}\right)$ (resp. $\left.\tilde{U}\left(M_{2}\right)\right)$ is the pressure at the point $M_{1}$ (resp. the displacement at the point $M_{2}$ ) in response to the external point force of the axisymmetric system (without the plate).

415 The frequency response of the velocity in the radial direction at the excitation point and the mean quadratic velocity on the outer surface of the shell are given in Fig. 8. They are given as a function of frequency between 100 and $600 \mathrm{~Hz}$ for the reference case of the whole system calculated by FEM and for the CTF method presented here. The upper frequency limit is set to $600 \mathrm{~Hz}$ in order to

${ }_{420}$ calculate the FEM reference solution more easily. The black dotted lines are the responses of the axisymmetric system (i.e. the stiffened shell without the plate) calculated by FEM. The blue dashed lines are the results of the CTF method that couples the stiffened shell described by CAA with the plate described by FEM. They are to compare with the references calculated by FEM and plotted in red solid lines. For the velocity plotted in Fig. 8 1 , a gray dotted line with cross markers represents the results of the CTF method where the stiffened shell admittances are calculated by FEM. It shows that this curve fits very well with the reference, whereas some differences can be seen with the CTF method using CAA. These differences can be explained by the fact that the CAA is, 430 as all numerical methods, only an approximation of the physical phenomena and can lead to slight deviations. Different parameters can be tuned to refine the results (as for example the maximum number of circumferential orders or the definition of the longitudinal wavenumber space) in exchange for higher computational costs. However, these results show that the CTF method is able to get close to the reference calculation and is thus validated for this case. 


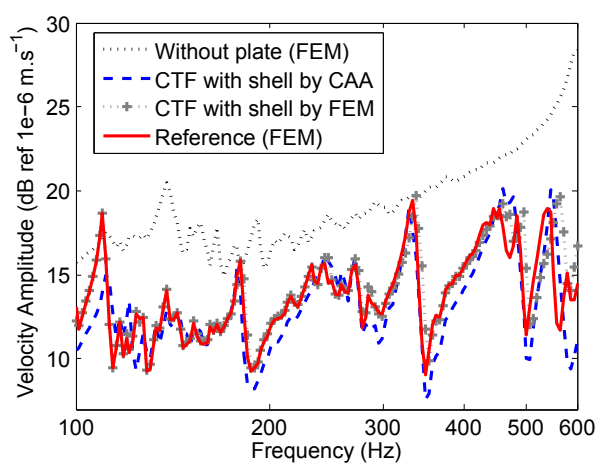

(a)

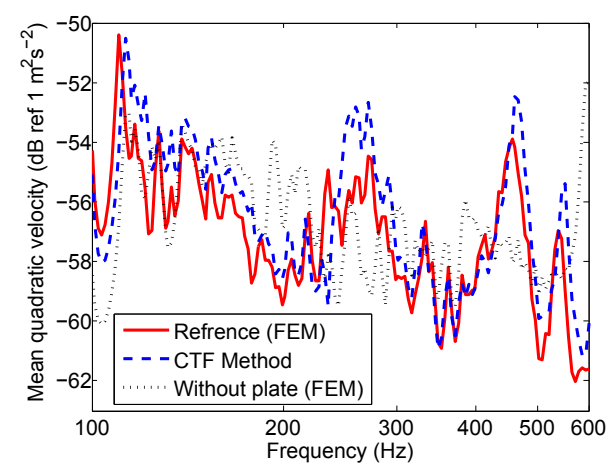

(b)

Figure 8: (a) Radial velocity amplitude ( $\mathrm{dB}$ ref $10^{-6} \mathrm{~m} \cdot \mathrm{s}^{-1}$ ) of the excitation point as a function of the frequency. (b) Mean quadratic velocity $\left(\mathrm{dB} r e f 1 \mathrm{~m}^{2} \cdot \mathrm{s}^{-2}\right)$ as a function of the frequency.

\subsection{Vibroacoustic behavior of a non-axisymmetric submerged shell \\ 4.4.1. Description of the system}

In this section, the CTF method is applied to a submerged test case. The case considered is shown in Fig. 9 and consists of a $5 \mathrm{~m}$ radius, $42.3 \mathrm{~m}$ length and $30 \mathrm{~mm}$ thick cylindrical shell stiffened with 51 stiffeners and 2 spherical bulkheads (10 mm thick, $30 \mathrm{~m}$ curvature radius). There are three different types of stiffeners and their spacing varies between 0.6 and $1 \mathrm{~m}$. The whole system is made of steel $(\eta=0.02)$ and is immersed in water. Clamped boundary conditions are used at the ends of the shell, $2 \mathrm{~m}$ away from the first and the last stiffeners. Non-axisymmetric internal frames are added between frames 13 and 19. They are composed of 6 identical curved plate, with the same dimensions as in section 4.3 (2 m long with a curvature radius of $4.8 \mathrm{~m}, 0.8 \mathrm{~m}$ wide and $15 \mathrm{~mm}$ thick). A point force is applied on frame 18 at the junction between the flange and the web. The plates and the excitation are chosen so that the system remains symmetric with regard to the plane $\theta=0$.

The only difference with the validation case presented in the previous subsection lies in the shell admittance calculation, which accounts, in the submerged case, for the heavy fluid loading. There are 12 line junctions, which are each divided in 10 segments, resulting in a set of 120 condensation functions. The admittances for a radial displacement in response to a radial force projected on these condensation functions are plotted in Fig. 10 at $f=1000 \mathrm{~Hz}$. In Fig. $10 \mathrm{a}$, the 12 diagonal blocks show the admittance amplitude when the displacements and forces are on the same junction, and have thus the biggest amplitudes. As there are 10 gate functions per junction, these blocks have a size of $10 \times 10$, and

460 one of them is circled in black. Every other block of the diagonals above and below the main one also has an important amplitude, due to the fact that the junctions are on the same T-cross-section stiffener. There are 10 of these extradiagonal blocks with a higher amplitude and one of them is circled in white. One 


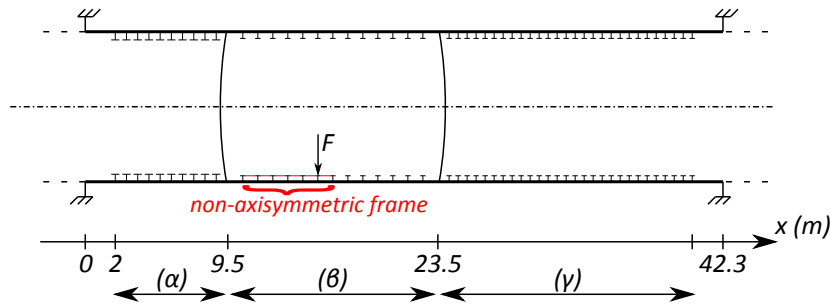

(a)

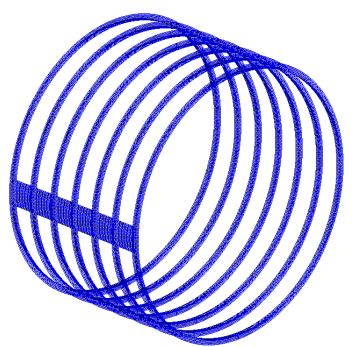

(b)

Figure 9: Submerged hull. Cylindrical shell: $5 \mathrm{~m}$ radius, $42.3 \mathrm{~m}$ length, $30 \mathrm{~mm}$ thick. Axisymmetric stiffeners: $(\alpha)$ spacing $0.75 \mathrm{~m}$, T-cross-section $(\mathrm{mm})$ : $300 \times 60 / 60 \times 300 ;(\beta)$ spacing $1 \mathrm{~m}$, T-cross-section $(\mathrm{mm}): 200 \times 15 / 15 \times 200 ;(\gamma)$ spacing $0.6 \mathrm{~m}$, T-cross-section $(\mathrm{mm})$ : $200 \times 25 / 15 \times 200$. (a) Section in the plane $\theta=0$. (b) Non-axisymmetric part (without the cylindrical shell).

can also see that the amplitude decreases with the distance to the excitation are uncoupled one from the other as there are only diagonal blocks linking two consecutive junctions.

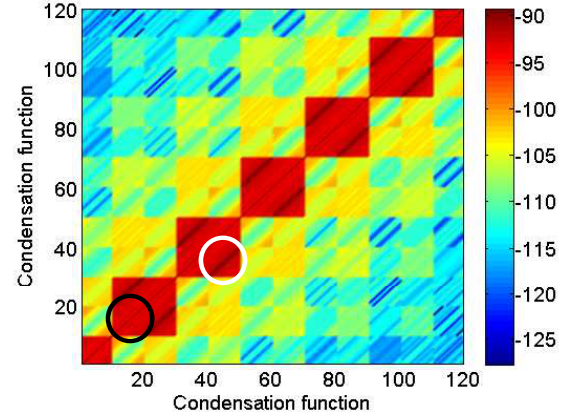

(a)

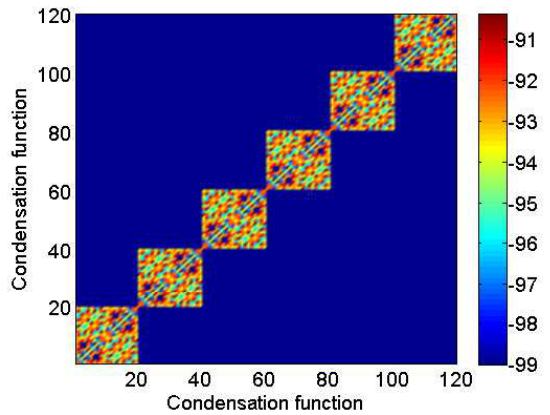

(b)

Figure 10: Amplitude of the admittance $\left(T_{r}, F_{r}\right)(\mathrm{dB}$ ref 1$)$ at $f=1000 \mathrm{~Hz}$ of the (a) axisymmetric shell (the black circle shows a diagonal block and the white circle shows an extradiagonal block) and the (b) non-axisymmetric internal frames.

\subsubsection{Results}

The mean quadratic velocity averaged on the outer surface of the shell is 470 plotted as a function of the frequency in Fig. 117. To describe properly the resonance peaks, 400 values are logarithmically spread over the frequency domain between 100 and $1000 \mathrm{~Hz}$. It shows that the non-axisymmetric internal frames have a weak influence on the mean quadratic velocity. Nevertheless, it can be 
said that a slight apparent damping effect appears, as predicted by the theory of fuzzy internals by Soize 34. The amplitude of the peaks of the mean quadratic velocity between 350 and $600 \mathrm{~Hz}$ is indeed lower in the non-axisymmetric case than in the axisymmetric case. The radiation efficiency $\sigma$ is plotted on Fig. 11b. It is defined as the ratio between the actual mean far-field radiated power $W_{a}$ and the power that would be radiated by a cylindrical shell of the same radiating surface $S$, having an uniform radial velocity $V_{0}$ :

$$
\sigma=\frac{W_{a}}{\rho_{e} c_{e} S V_{0}^{2}}
$$

for $\rho_{e}=1000 \mathrm{~kg} \cdot \mathrm{m}^{-3}$ the fluid density and $c_{e}=1500 \mathrm{~m} \cdot \mathrm{s}^{-1}$ the speed of sound in water.

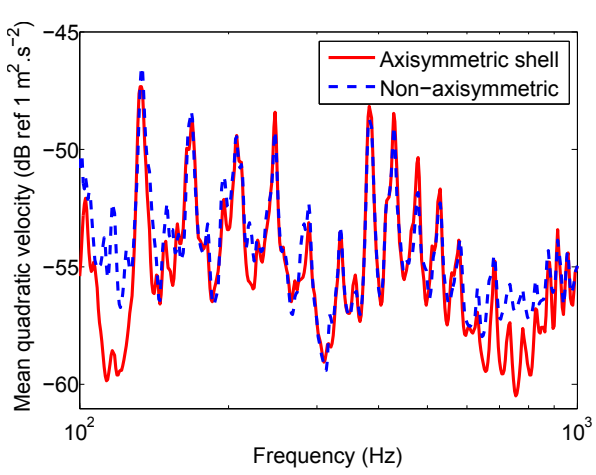

(a)

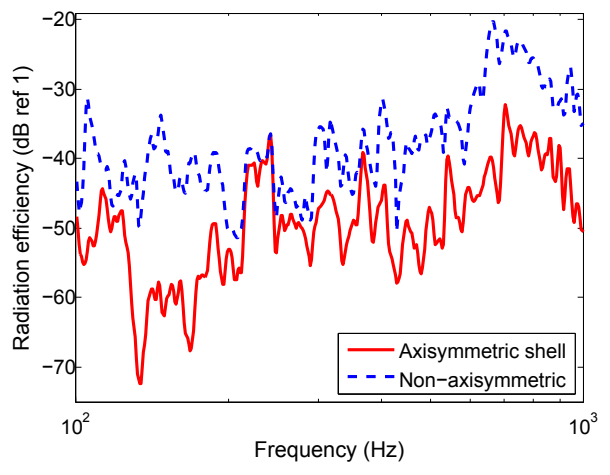

(b)

Figure 11: Frequency responses of the system excited by a radial point force, with and without the non-axisymmetry: (a) Amplitude ( $\mathrm{dB}$ ref $1 \mathrm{~m}^{2} \cdot \mathrm{s}^{-2}$ ) of the mean quadratic velocity on the outer surface of the shell. (b) Radiation efficiency (dB ref 1 ).

To explain these differences in the radiation efficiency, although the mean quadratic velocity is only slightly changed, a wavenumber analysis is done 35. The shell radial acceleration level at $f=1000 \mathrm{~Hz}$ is plotted in the wavenumber space in Fig. 12 for the two configurations (with and without the plates). As the problem is symmetrical, the variables are even functions of the circumferential orders and thus only the positive circumferential orders have been plotted. The white line shows the radiation circle (elliptical in Fig. $12 \mathrm{a}$ and c due to the plotting aspect ratio), defined by:

$$
\left(\frac{k_{\theta}}{R}\right)^{2}+k_{x}^{2}=\left(\frac{2 \pi f}{c_{e}}\right)^{2}
$$

The radiation circle represents the supersonic acoustic domain, in which the components radiate to the far-field for a flat plate. The radiation circle remains also a good indicator for localizing the radiating waves of a shell having a low 
curvature. On the overall plot, it can be seen that adding the plates has little influence on the highest values of the acceleration spectrum in the wavenumber space (in red in Fig. $12 \mathrm{k}, \mathrm{c}$ ). This indicates that the most contributing waves in terms of the vibration energy propagation on the shell are globally the same for the two cases. It results in few changes on the mean quadratic velocity, as shown in Fig. 11a. Nevertheless, when zooming in on the radiation circle, 500 one can observe that the components (which amplitudes are clearly lower than the highest values outside the circle) are significantly influenced by the plates (see Fig. $12 \mathrm{~b}, \mathrm{~d}$ ). As these components radiate sound efficiently, it explains the difference in the radiation efficiency observed in Fig. 11b. In general, an axisymmetric shell is not an efficient radiator, but it can be seen that breaking the axisymmetry by adding 3D internal structures increases the radiation efficiency. This trend can be explained by the fact that the non-axisymmetric frames couple circumferential orders together. These results are consistent with the literature [17, 21].

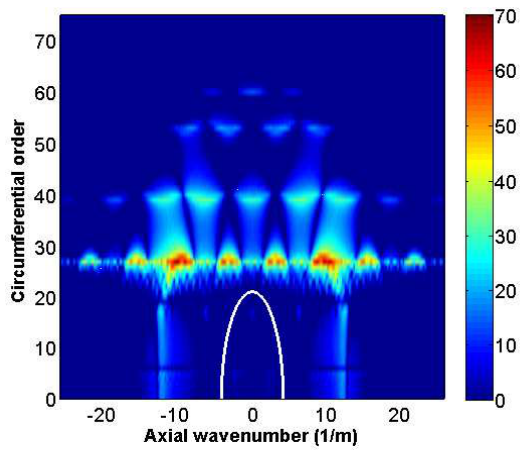

(a)

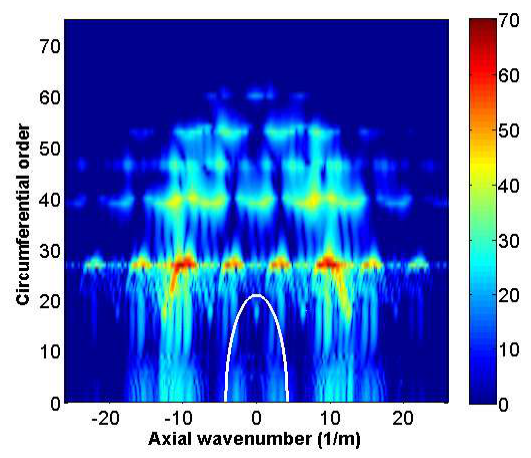

(c)

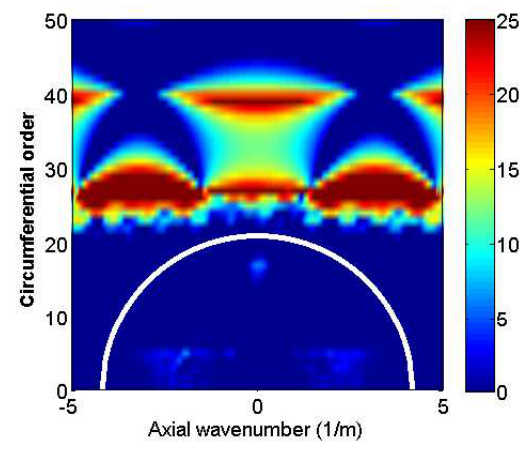

(b)

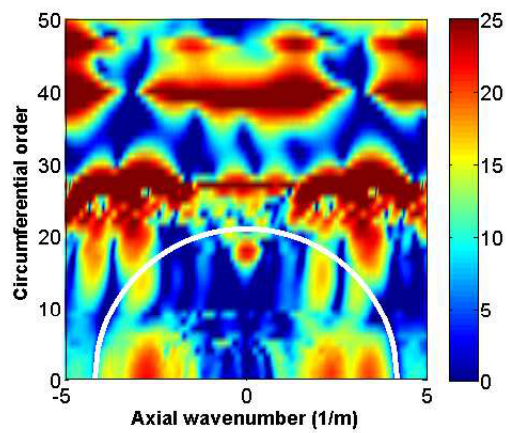

(d)

Figure 12: Radial acceleration of the shell $\left(\mathrm{dB}\right.$ ref $\left.10^{-6} \mathrm{~g}\right)$ in the wavenumber space under a radial unitary force excitation at $1000 \mathrm{~Hz}$. (a) Axisymmetric shell. (b) Axisymmetric shell (zoom on the radiation circle). (c) Non-axisymmetric shell. (d) Non-axisymmetric shell (zoom on the radiation circle). 
The radial displacements for the submerged stiffened shell, with and without added plates, are presented in Fig. 13 The results are given at $1000 \mathrm{~Hz}$ and the values are normalized to the highest displacement amplitude for each plot. In Fig. 13a and Fig. 13p, the excitation is radial while in Fig. 13p and Fig. $13 \mathrm{~d}$ the excitation is in the longitudinal direction. One can see that adding non-axisymmetric internal frames modifies the vibroacoustic behavior of the 515 stiffened submerged shell. More particularly in this case, the vibrational energy propagates easily in the longitudinal direction due to the strong stiffness of the plates in-plane motion, and is transmitted to the connected stiffeners. Thus, the high displacements are spread further from the point load in the non-axisymmetric cases, especially when the excitation is longitudinal.

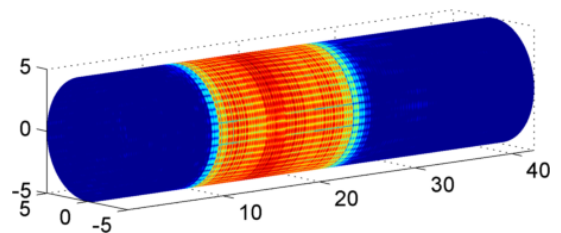

(a)

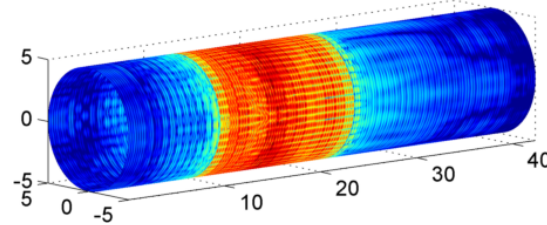

(c)

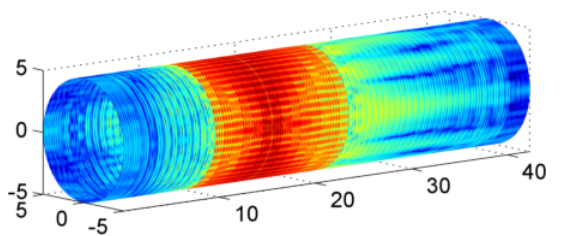

(b)

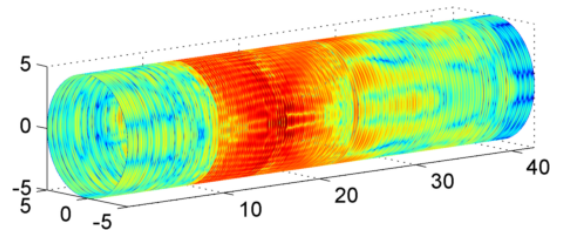

(d)

Figure 13: Radial displacements level (dB ref $10^{-6} \mathrm{~g}$ ) normed to the maximum value (for each load case) of the cylinder to a harmonic unitary force excitation at $1000 \mathrm{~Hz}$. (a) Axisymmetric shell under radial excitation. (b) Axisymmetric shell under longitudinal excitation. (c) Nonaxisymmetric shell under radial excitation. (d) Non-axisymmetric shell under longitudinal excitation.

In Fig. 14, the far-field sound pressure level at $100 \mathrm{~m}$ is plotted at $1000 \mathrm{~Hz}$ for the axisymmetric and non-axisymmetric shell. The same color scale is used for the two subplots. The shell is oriented in the same way as in the representation of the shell displacements in Fig. 13. First, consistently with Fig. 11, it is clear that the radiated sound level is higher for the non-axisymmetric case. Then, because of the coupling of different circumferential orders, it can be said that the non-axisymmetric internal frames alter the directivity pattern by increasing its complexity.

From a computational point of view, adding these non-axisymmetric internal frames lengthen the calculation by about $60 \%$ in comparison with the axisym530 metric stiffened shell. Assuming that the frames admittances used in CAA were already stored in a database and did not need to be calculated again, one frequency for the axisymmetric case was performed in about 75 seconds. Adding 


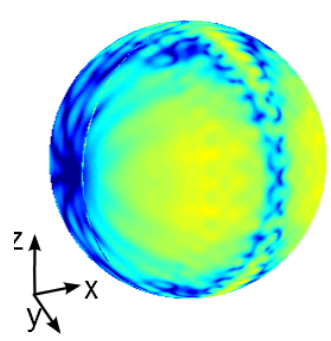

(a)

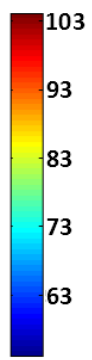

stis

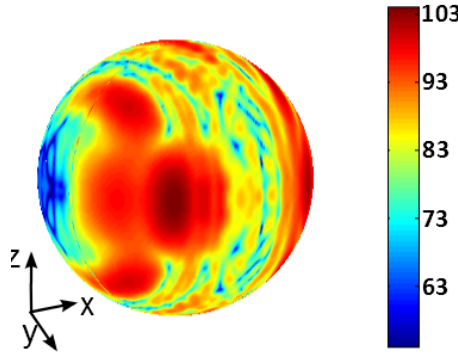

(b)

Figure 14: 3D representation of the far-field sound pressure level at $100 \mathrm{~m}\left(\mathrm{~dB}\right.$ ref $\left.10^{-6} \mathrm{~Pa}\right)$ of the cylinder to a radial harmonic unitary force excitation at $f=1000 \mathrm{~Hz}$. (a) Axisymmetric shell. (b) Non-axisymmetric shell.

the non-axisymmetric internal frames represents thus a difference of about 45 seconds for each frequency of calculation on the computer used for the tests (24 Gb RAM, 6 core processors at $2.66 \mathrm{GHz}$ ). It is worth noting that the calculation time for the admittances of the non-axisymmetric internal frame is short as modal superposition with residual modes is used. It is hard to compare these results to some other numerical methods, because very few of them are able to predict the behavior of a submerged hull in the considered frequency range,

${ }_{540}$ but it can be said that the computational time remains acceptable for practical applications.

\section{Conclusions}

A sub-structuring method has been proposed to couple subsystems along lines by condensing transfer functions. These transfer functions are calculated ${ }_{545}$ for each uncoupled subsystem separately. Different methods can be used depending on the characteristics of the subsystem, which gives great flexibility of the method. Once the condensed transfer functions calculated, a linear system is solved to deduce the coupling forces at the junctions. Finally, the response at any point of the considered coupled system can be calculated.

In this paper, three different condensation functions have been presented and validated on a basic test case made of two coupled plates. Moreover, CTF calculations using the gate functions have been used to couple together a numerical model of an in vacuo axisymmetric stiffened shell and finite element models of the non-axisymmetric internal frames. It has shown good agreement 555 with FEM results for this complex test case. The proposed method has then been applied to study the influence of non-axisymmetric internal frames on the vibroacoustic behavior of a submerged stiffened shell.

This last example illustrates well the interest of this approach for coupling different numerical models (i.e. CAA with FEM) and for taking the fluid loading on the cylindrical shell into account easily. From these results, as non- 
axisymmetries tend to couple circumferential orders together, it can be said that including non-axisymmetric internal frames can alter greatly the vibroacoustic behavior of the shell. On the example of plates linking adjacent stiffeners, it is clear that they play a key role as they tend to increase the radiation efficiency of the system and can alter the vibrational and acoustic energy distribution.

More generally, the present approach can be used to partition vibroacoustic problems into several subsystems coupled along lines, and can be applied to a wide range of practical problems. Another advantage of this approach is the ability to quickly reanalyze subsystems for optimization. For instance, if one 570 of the subsystems properties is modified, it is not necessary to recalculate the condensed transfer functions of the other subsystems. Computation time can thus be saved by reusing a database of condensed transfer functions.

\section{Appendix A. Calculation of condensed transfer functions by FEM}

To calculate the condensed transfer functions matrix and the free displacement vectors of the isolated subsystems, the harmonic responses to the force corresponding to the condensation functions on the coupling junction and to external forces must be calculated. When a subsystem is modeled by FEM, different methods can be used to calculate a frequency response. Three of them have been tested and are recalled in this section.

Direct calculation: It extracts the mass and stiffness matrices, respectively $\mathbf{M}$ and $\mathbf{K}$, from the FE model and introduces structural damping $\eta$ through a complex stiffness value $\mathbf{K}^{*}=\mathbf{K}(1+\jmath \eta)$. At a given angular frequency $\omega$, the displacement amplitude $\mathbf{U}_{\mathbf{0}}$ is calculated from the dynamics equation in the frequency domain :

$$
\mathbf{U}_{\mathbf{0}}=\left(-\omega^{2} \mathbf{M}+\mathbf{K}^{*}\right)^{-1} \mathbf{F}_{\mathbf{0}}
$$

The direct calculation gives accurate results but is time consuming.

Modal superposition: It solves the eigenvalue problem of the conservative dynamic system to build a basis of mode shapes $\boldsymbol{\Phi}_{\mathbf{n}}$ :

$$
\omega_{n}^{2} \mathbf{M} \boldsymbol{\Phi}_{\mathbf{n}}=\mathbf{K} \boldsymbol{\Phi}_{\mathbf{n}}
$$

Physically speaking, $\omega_{n}$ represent the eigenfrequencies of the system while $\boldsymbol{\Phi}_{\mathbf{n}}$ are the associated mode shapes. The basis is theoretically of infinite dimension but for obvious practical reasons it must be truncated to an integer $N_{m}$. The frequency response is then a linear combination of these modes:

$$
\mathbf{U}_{\mathbf{0}}=\sum_{n=1}^{N_{m}} a_{n} \boldsymbol{\Phi}_{\mathbf{n}}
$$


where $a_{n}$ is called the modal coefficient and is calculated from the reduced mass and stiffness matrices, $\underline{\mathbf{M}}=\boldsymbol{\Phi}^{\mathrm{T}} \mathbf{M} \boldsymbol{\Phi}$ and $\underline{\mathbf{K}}=\boldsymbol{\Phi}^{\mathrm{T}} \mathbf{K} \boldsymbol{\Phi}$ and the reduced force $\underline{\mathbf{F}}=\boldsymbol{\Phi}^{\mathrm{T}} \mathbf{F}$ :

$$
a_{n}=\left(\underline{\mathbf{K}}^{*}-\omega^{2} \underline{\mathbf{M}}\right)^{-1} \underline{\mathbf{F}}
$$

The damping can be introduced in the reduced stiffness matrix: $\underline{\mathbf{K}}^{*}=$ $\underline{\mathbf{K}}(1+\jmath \eta)$. Comparing to the direct calculation, this method is much faster but has a poor convergence in some frequency bands (i.e. outside of resonance peaks), because of the basis truncation.

Modal superposition + residual modes: A quasi-static mode shape $\boldsymbol{\Psi}$ (i.e. a particular response at a very low frequency $\omega_{0}$ ) is calculated as follow:

$$
\boldsymbol{\Psi}=\left(\mathbf{K}-\omega_{0}^{2} \mathbf{M}\right)^{-1} \mathbf{F}_{\mathbf{0}}
$$

It is then added to the modal basis to define a new basis $\mathbf{P}=\left\{\boldsymbol{\Phi}_{\mathbf{n}} \mathbf{\Psi}\right\}$ and to compensate the effect of the truncation. It is worth noting that the basis $\mathbf{P}$ is generally not orthogonal and needs to be diagonalized. To do so, the following eigenvalue problem is solved:

$$
\left(\underline{\mathbf{K}}-\underline{\omega}_{n}^{2} \underline{\mathbf{M}}\right) \mathbf{Q}=0
$$

with $\underline{\mathbf{M}}=\mathbf{P}^{\mathrm{T}} \mathbf{M P}$ and $\underline{\mathbf{K}}=\mathbf{P}^{\mathrm{T}} \mathbf{K} \mathbf{P}$. As $\left(\underline{\mathbf{K}}-\underline{\omega}_{n}^{2} \underline{\mathbf{M}}\right)$ is a symmetric semi-definite matrix, the eigenvectors $\mathbf{Q}$ are orthogonal. One defines new reduced mass and stiffness matrices:

$$
\left\{\begin{array}{l}
\underline{\underline{\mathbf{M}}}=\mathbf{Q}^{\mathrm{T}} \underline{\mathbf{M}} \mathbf{Q} \\
\underline{\underline{\mathbf{K}}}=\mathbf{Q}^{\mathrm{T}} \underline{\mathbf{K}} \mathbf{Q}
\end{array}\right.
$$

along with a new reduced force vector $\mathbf{F}=\mathbf{Q}^{\mathrm{T}} \mathbf{P}^{\mathrm{T}} \mathbf{F}_{\mathbf{0}}$. Introducing again damping in the reduced stiffness matrix, one deduces the displacement through the following equations:

$$
\mathbf{U}_{\mathbf{0}}=\sum_{n=1}^{N_{m}} a_{n}(\mathbf{P Q})_{\mathbf{n}}
$$

with

$$
a_{n}=\left(\underline{\underline{\mathbf{K}}}^{*}-\omega^{2} \underline{\underline{\mathbf{M}}}\right)^{-1} \underline{\underline{\mathbf{F}}}
$$

Comparing to the previous alternative, the convergence is greatly increased and the calculation time remains low. 


\section{Acknowledgment}

This work was funded by DCNS and performed within the framework of the LabEx CeLyA of Université de Lyon, operated by the French National Research Agency (ANR-10-LABX-0060/ANR-11-IDEX-0007).

\section{References}

[1] L. Maxit, J.-M. Ginoux, Prediction of the vibro-acoustic behavior of a submerged shell non periodically stiffened by internal frames, The Journal of the Acoustical Society of America 128 (1) (2010) 137-151.

[2] F. A. Firestone, The mobility method of computing the vibration of linear mechanical and acoustical systems: Mechanical-electrical analogies, Journal of applied Physics 9 (6) (1938) 373-387.

[3] E. L. Hixson, Mechanical impedance, Shock and vibration handbook (1976) $10-1$.

[4] M. R. Peterson, D. E. Boyd, Free vibrations of circular cylinders with longitudinal, interior partitions, Journal of Sound and Vibration 60 (1) (1978) 45-62.

[5] R. S. Langley, A dynamic stiffness technique for the vibration analysis of stiffened shell structures, Journal of Sound and Vibration 156 (3) (1992) 521-540.

[6] J. Missaoui, L. Cheng, Vibroacoustic analysis of a finite cylindrical shell with internal floor partition, Journal of Sound and Vibration 226 (1) (1999) $101-123$.

[7] Y.-S. Lee, M.-H. Choi, Free vibrations of circular cylindrical shells with an interior plate using the receptance method, Journal of Sound and Vibration 248 (3) (2001) 477-497.

[8] Z. Zhao, M. Sheng, Y. Yang, Vibration transmission of a cylindrical shell with an interior rectangular plate with the receptance method, Advances in Acoustics and Vibration 2012.

[9] E. Rebillard, B. Laulagnet, J.-L. Guyader, Influence of an embarked springmass system and defects on the acoustical radiation of a cylindrical shell, Applied Acoustics 36 (2) (1992) 87-106.

[10] J. D. Achenbach, J. Bjarnason, T. Igusa, Effect of a vibrating substructure on acoustic radiation from a cylindrical shell, Journal of Vibration and Acoustics 114 (3) (1992) 312-318.

[11] J. Bjarnason, J. D. Achenbach, T. Igusa, Acoustic radiation from a cylindrical shell with an internal plate, Wave Motion 15 (1) (1992) 23-41. 
[12] S.-H. Choi, J. D. Achenbach, T. Igusa, The effect of periodically attached substructures on the excitation of submerged cylindrical shells, Journal of Sound and Vibration 177 (3) (1994) 379-392.

[13] S.-H. Choi, T. Igusa, J. D. Achenbach, Nonaxisymmetric vibration and acoustic radiation of a submerged cylindrical shell of finite length containing internal substructures, The Journal of the Acoustical Society of America 98 (1) (1995) 353-362.

[14] S.-H. Choi, T. Igusa, J. D. Achenbach, Acoustic radiation from a finitelength shell with non-axisymmetric substructures using a surface variational principle, Journal of Sound and Vibration 197 (3) (1996) 329-350.

[15] B. Laulagnet, J.-L. Guyader, Sound radiation from finite cylindrical shells, partially covered with longitudinal strips of compliant layer, Journal of Sound and Vibration 186 (5) (1995) 723-742.

[16] J. M. Cuschieri, D. Feit, Influence of circumferential partial coating on the acoustic radiation from a fluid-loaded shell, The Journal of the Acoustical Society of America 107 (6) (2000) 3196-3207.

[17] D. M. Photiadis, B. H. Houston, E. G. Williams, J. A. Bucaro, Resonant response of complex shell structures, The Journal of the Acoustical Society of America 108 (3) (2000) 1027-1035.

[18] D. M. Photiadis, J. A. Bucaro, B. H. Houston, The effect of internal oscillators on the acoustic response of a submerged shell, The Journal of the Acoustical Society of America 101 (2) (1997) 895-899.

[19] J. A. Bucaro, A. J. Romano, A. Sarkissian, D. M. Photiadis, B. H. Houston, Local admittance model for acoustic scattering from a cylindrical shell with many internal oscillators, The Journal of the Acoustical Society of America 103 (4) (1998) 1867-1873.

[20] G. Maidanik, Response of ribbed panels to reverberant acoustic fields, the Journal of the Acoustical Society of America 34 (6) (1962) 809-826.

[21] M. H. Marcus, B. H. Houston, The effect of internal point masses on the radiation of a ribbed cylindrical shell, The Journal of the Acoustical Society of America 112 (3) (2002) 961-965.

[22] S. Sadiq, Submerged double cylinder multi-excitation analysis through linear superposition method with FEM-BEM experimental approach, in: Proceedings of International Bhurban Conference on Applied Sciences \& Technology, Islamabad, Pakistan, 2010.

[23] H. Peters, R. Kinns, N. J. Kessissoglou, Effects of internal mass distribution and its isolation on the acoustic characteristics of a submerged hull, Journal of Sound and Vibration 333 (6) (2014) 1684-1697. 
[24] M. M. Ettouney, R. P. Daddazio, N. N. Abboud, The interaction of a submerged axisymmetric shell and three-dimensional internal systems, International Journal for Numerical Methods in Engineering 37 (17) (1994) 2951-2970.

[25] M. C. C. Bampton, R. R. Craig Jr, Coupling of substructures for dynamic analyses., AIAA Journal 6 (7) (1968) 1313-1319.

[26] G. M. L. Gladwell, Branch mode analysis of vibrating systems, Journal of Sound and Vibration 1 (1) (1964) 41-59.

[27] C. Lesueur, Rayonnement acoustique des structures: vibroacoustique, interactions fluide-structure (In French), Eyrolles, 1988.

[28] S. Rubin, Improved component-mode representation for structural dynamic analysis, AIAA journal 13 (8) (1975) 995-1006.

[29] B. Fornberg, A practical guide to pseudospectral methods, Vol. 1, Cambridge university press, 1998.

[30] V. Meyer, L. Maxit, J.-L. Guyader, T. Leissing, C. Audoly, A condensed transfer function method as a tool for solving vibroacoustic problems, NOVEM 2015, Dubrovnik, Croatia, 2015.

[31] A. Harari, B. E. Sandman, Radiation and vibrational properties of submerged stiffened cylindrical shells, The Journal of the Acoustical Society of America 88 (4) (1990) 1817-1830.

[32] W. Flügge, Statik und dynamik der schalen, Springer, 1962.

[33] A. W. Leissa, Vibration of shells, Vol. 288, Scientific and Technical Information Office, National Aeronautics and Space Administration Washington, DC, USA, 1973.

[34] C. Soize, Probabilistic structural modeling in linear dynamic analysis of complex mechanical systems, I-theoretical elements, La Recherche Aérospatiale (English edition) 5 (1986) Pages-23.

[35] E. G. Williams, B. H. Houston, J. A. Bucaro, Experimental investigation of the wave propagation on a point-driven, submerged capped cylinder using k-space analysis, The Journal of the Acoustical Society of America 87 (2) (1990) 513-522. 